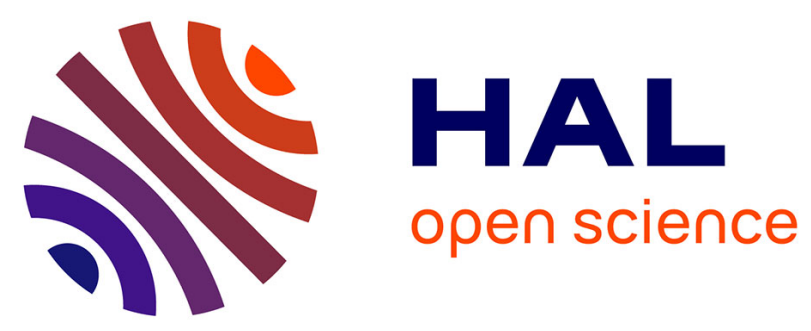

\title{
In vivo surface dosimetry with a scintillating fiber dosimeter in preclinical image-guided radiotherapy
}

Coralie Le Deroff, Elodie A. Pérès, Xavier Ledoux, Toutain Jérôme, Anne-Marie Frelin-Labalme

\section{- To cite this version:}

Coralie Le Deroff, Elodie A. Pérès, Xavier Ledoux, Toutain Jérôme, Anne-Marie Frelin-Labalme. In vivo surface dosimetry with a scintillating fiber dosimeter in preclinical image-guided radiotherapy. Medical Physics, 2020, 47 (1), pp.234-241. 10.1002/mp.13903 . hal-02363478

HAL Id: hal-02363478

https://hal-normandie-univ.archives-ouvertes.fr/hal-02363478

Submitted on 11 Feb 2020

HAL is a multi-disciplinary open access archive for the deposit and dissemination of scientific research documents, whether they are published or not. The documents may come from teaching and research institutions in France or abroad, or from public or private research centers.
L'archive ouverte pluridisciplinaire HAL, est destinée au dépôt et à la diffusion de documents scientifiques de niveau recherche, publiés ou non, émanant des établissements d'enseignement et de recherche français ou étrangers, des laboratoires publics ou privés. 


\section{In vivo surface dosimetry with a scintillating fiber dosimeter in preclinical image-guided radiotherapy}

Short title: Small animal in vivo dosimetry

Coralie Le Deroff ${ }^{1}$, Ph.D., Elodie A. Pérès ${ }^{2}$, Ph.D., Xavier Ledoux ${ }^{1}$, Ph.D., Jérôme Toutain ${ }^{2}$, M.S. and Anne-Marie Frelin-Labalme ${ }^{1,3}$, Ph.D.,

${ }^{1}$ Grand Accélérateur National d'Ions Lourds (GANIL), CEA/DRF-CNRS/IN2P3, Boulevard Henri Becquerel, 14076 Caen, France

${ }^{2}$ Normandie Univ, UNICAEN, CEA, CNRS, ISTCT/CERVOxy group, Caen, France

${ }^{3}$ Advanced Resource Centre for Hadrontherapy in Europe (ARCHADE) Program, Caen, France

\section{Corresponding author:}

Dr Anne-Marie Frelin-Labalme, Grand Accélérateur National d'Ions Lourds (GANIL),

CEA/DRFCNRS/IN2P3, Boulevard Henri Becquerel, 14076 Caen, France, phone: 3323145 45 30. anne-marie.frelin@ @anil.fr 


\title{
In vivo surface dosimetry with a scintillating fiber dosimeter in preclinical image-guided radiotherapy
}

\begin{abstract}
Purpose. New preclinical image-guided irradiators and treatment planning systems represent a huge progress in radiobiology. Nevertheless, quality control of preclinical treatments is not as advance as in clinical radiotherapy and in vivo dosimetry is little developed. In this study, we evaluate the use of a scintillating fiber dosimeter called DosiRat to verify the agreement between the doses planned with SmART-Plan and the measured doses during small animal irradiations. Methods. In vivo dosimetry was first evaluated with DosiRat through dose measurements performed at the surface of a $3 \times 9 \times 3 \mathrm{~cm}^{3}$ phantom. Measured and planned doses were compared for different irradiation conditions (prescription point, anterior and posterior beams, $5 \mathrm{~mm}$ and $10 \mathrm{~mm}$ irradiation fields). In a second phase, measured and planned doses were compared for rat brain irradiations performed with anterior beams, with DosiRat positioned at the beam entrance. Comparisons were performed for different tube currents (1.3 and $13 \mathrm{~mA}$ ), collimations (5, 10 and $25 \mathrm{~mm}$ diameter) and planned doses (0.1, 0.5, 2 and $10 \mathrm{~Gy})$. Results. In the case of the phantom irradiations, planned and measured doses showed discrepancies smaller than the $5 \%$ accuracy of the TPS, except in cases in which the dosimeter was not centered in the irradiation field. The differences were larger for animal irradiations (from $-3.3 \%$ to $8.8 \%$ ) because of variations of the beam energy spectrum and the non-equivalence between materials at medium and low energy. Conclusions. This study highlighted the complexity to implement one-dimension in vivo dosimetry in orthovoltage millimetric beams. Nevertheless, DosiRat is well adapted to in vivo dosimetry because of its small volume and its direct reading and allowed in vivo control of planned doses for anterior beams down to $5 \mathrm{~mm}$ diameter.
\end{abstract}


Key words: Preclinical radiotherapy, scintillating fiber dosimeter, in vivo dosimetry 


\section{Introduction}

The use of preclinical image-guided irradiators is rapidly spreading due to their strong similarities with clinical systems on a smaller scale. ${ }^{1}$ New systems such as the X-RAD $225 C x$, now sold as the X-RAD SmART (Precision X-Ray, Inc., North Branford, CT, USA) and the Small Animal Radiation Research Platform (SARRP, Xstrahl Inc., Camberley, Surrey, UK) irradiators use dedicated treatment planning softwares (TPS) which provides more robust dosimetry and treatment panning accuracy for preclinical studies that quantify biological responses to ionizing radiations. ${ }^{2}$ Nevertheless, the quality control of preclinical treatments is not as advanced as in clinical practice ${ }^{3,4}$ and preclinical in vivo dosimetry is little developed ${ }^{5,6}$. In clinical radiotherapy, in vivo dosimetry verifies the agreement between planned and delivered doses, which allows the detection of systematic errors coming from various origins (beam dose calibration, accelerator instabilities, patient positioning...). Preclinical radiotherapy presents even more sources of error than clinical radiotherapy. The operation of the irradiator can lead to human error during the change of the external filtration between imaging and irradiation, or during the collimator selection. Misalignment of the beam or the stage can also occurs as well as animal motion during irradiation. All these events have a significant impact on dose accuracy and consequently on the interpretation of radiobiological results. An accurate control of delivered dose is thus of major importance to improve the reproducibility and reliability of preclinical investigations.

Several teams carried out studies to answer the need of in vivo dosimetry. Kuess et al used thermoluminescent dosimeters (TLD) inserted in the animal mouth during irradiation. ${ }^{7}$ They showed satisfying results, with measurements comparable to reference measurements performed in a phantom. Nevertheless, even if a careful calibration and handling of TLDs can provide an accuracy between $1 \%$ and $3 \%,{ }^{8}$ their manipulation is quite difficult and their read-out is delayed. Ngwa et al used metal oxide semiconductor field effect transistors (MOSFET) dosimeters surgically implanted into animals to assess the effect of tissue heterogeneity on dose calculation for different anatomical locations. ${ }^{5}$ They reported that MOSFESTs can perform real-time measurements and could serve as fiducial markers. Nevertheless, they also pointed out the energy dependence and angular dependence of these detectors. Anvari et al utilized the electronic portal imaging device (EPID) on the SARRP for quality assurance measurements 
with potential for usage in in vivo dosimetry. ${ }^{6}$ Granton et al also studied this approach. They showed that the imaging panel could be used to verify the agreement between planned and delivered doses with a $5 \%$ uncertainty for beams as small as $4 \mathrm{~mm} .^{9}$

Other dosimeters adapted to millimeter beams and medium energy X-rays can be considered to perform small animal in vivo dosimetry. Among them, semiconductor diodes and diamond detectors have the advantage of their small size. Scintillating fiber dosimeters (SFD) are also good candidates to perform real time controls in preclinical dosimetry. Their sensitive volume is made of plastic scintillating fibers (usually polystyrene or polyvinyltoluene based) of small diameter (0.5 to $1 \mathrm{~mm}$ ). SFDs have been extensively studied for dosimetry in clinical radiotherapy because of their water equivalence at high energy (MeV X-rays), their excellent reproducibility and response linearity with dose and dose rate. ${ }^{10,11}$ Their suitability for small field dosimetry has already been demonstrated in clinical radiotherapy ${ }^{12-14}$ and more recently in preclinical radiotherapy. ${ }^{15}$ Because of all these characteristics, PSDs are a very promising tool for in vivo dosimetry to control the delivered dose as well as potential misalignments between the target and the beam. We previously developed and evaluated a prototype named DosiRat, specifically adapted to preclinical millimeter and orthovoltage beams. ${ }^{16,17}$ This dosimeter showed a highly repeatable, reproducible and linear response with dose and dose rate in treatment beams. ${ }^{9}$ It was also successfully used in preclinical relative dosimetry to measure percentage depth dose distributions in a $4 \times 4 \mathrm{~cm}^{2}$ irradiation field and to measure relative output factors at the surface of a phantom for irradiation fields as small as $2.5 \mathrm{~mm}$ diameter. ${ }^{10}$ In this study, we evaluate the ability of DosiRat to perform in vivo dose control during phantom and rat brain irradiations. Contrary to other preclinical studies where the objective was to assess the effect of tissue heterogeneity and composition on treatment plans, ${ }^{5,7}$ our main purpose is to verify that the delivered dose corresponds to the intended one and that it does not suffer from setup errors. Consequently, non-invasive surface measurements have been preferred. 


\section{Materials and methods}

The response of the scintillating fiber dosimeter for in vivo dosimetry was evaluated by comparing DosiRat measurements to planned doses in two configurations: a single beam irradiation on a rectangular polymethyl methacrylate (PMMA) phantom, which dimensions $\left(3 \times 9 \times 3 \mathrm{~cm}^{3}\right)$ are representative of animal size, and during rat brain irradiation.

\subsection{Image-guided preclinical irradiator}

This study was performed with the X-RAD $225 \mathrm{Cx}$ preclinical irradiator. This platform principally consists of a $225 \mathrm{kVp}$ X-ray tube mounted on a rotating C-arm, associated to a high resolution imaging panel (Perkin Elmer, Fremont, California, USA) and a precise positioning stage. The X-ray beam can be used either for imaging acquisitions or for treatment irradiations. Imaging acquisitions usually use tube voltages between 40 and $120 \mathrm{kVp}$ and tube currents below $1 \mathrm{~mA}$ with an external filtration of $2 \mathrm{~mm}$ of aluminum. Two acquisition modes are supported by the system: a fluoroscopy mode, providing real time projections in two dimensions, and a Cone Beam Computed Tomography (CBCT) mode, providing 3-dimensionnal reconstructions with voxel sizes of 100, 200 or $400 \mu \mathrm{m}$. Treatment irradiations generally use a tube voltage of $225 \mathrm{kVp}$ and a tube current of $13 \mathrm{~mA}$ with an external filtration of $0.3 \mathrm{~mm}$ of copper. Various collimators can be used depending on the target volume. In this study, we worked with circular collimators of diameters ranging from 5 to $25 \mathrm{~mm}$ and a square collimator of $4 \times 4 \mathrm{~cm}^{2}$.

2.1.1. Absolute dose rate. The irradiator absolute dose rate was measured at $225 \mathrm{kVp}$ and $13 \mathrm{~mA}$ with a PTW 30013 ionization chamber calibrated in absorbed dose to water for various beam qualities. These calibrations are traceable to the standards of the German National Laboratory Physikalisch-Technische Bundesanstalt (PTB, Braunschweig, Germany) and were interpolated for our irradiator beam quality. According to the IAEA TRS-398 protocol for orthovoltage beams, the measurement was performed at isocenter (corresponding to a source-to-detector distance [SDD] of $30.7 \mathrm{~cm}$ ) at a depth of $2 \mathrm{~cm}$ in a water tank of $30 \times 30 \times 30 \mathrm{~cm}^{3}$ positioned above the imaging panel on a customized mechanical support. The TRS-398 protocol was slightly modified by using a $4 \times 4 \mathrm{~cm}^{2}$ irradiation field instead of 
$10 \times 10 \mathrm{~cm}^{2}$, following preclinical practice. ${ }^{18}$ The average value of 3 ionization chamber readings $(M)$ was converted into absorbed dose to water $(D)$ following Eq. (1):

$$
D=N_{w} k_{Q} k_{T P} k_{\text {ion }} k_{\text {pol }} M
$$

with $N_{w}$ the ionization chamber calibration factor and $k_{Q}$ the beam quality correction factor interpolating the closest specified correction factors given by the calibration certificates. $k_{T P}, k_{i o n}$ and $k_{p o l}$ are the temperature-pressure, ion collection efficiency and polarity correction factors respectively and have been experimentally determined.

2.1.2. Ramp-up effect. The absolute dosimetry was performed with a beam stabilized in voltage and current. Nevertheless, a ramp-up time exists to reach the nominal tube voltage and current, during which the dose rate is lower than the stabilized one. This ramp-up time was measured in order to correct, if needed, the exposure time and avoid under-dose deposition.

Air kerma was measured at the irradiator isocenter with the PTW 30013 ionization chamber calibrated in air kerma for two different tube currents: 1.3 and $13 \mathrm{~mA}$. Measurements were performed for irradiation times of 5,10,15,30,60,120 and 180 seconds and the ramp-up time was deduced from the intercept of the air kerma as a function of irradiation time with the time-axis.

\subsection{Scintillating fiber dosimetry}

The dose measurements were performed with DosiRat scintillating fiber dosimeter, previously fully described by Le Deroff et al. ${ }^{16,17}$ In all this study, we used a BCF-12 (polystyrene based, Saint-Gobain Crystal, Paris, France) scintillating probe of $2 \mathrm{~mm}$ length and $1 \mathrm{~mm}$ diameter, presenting an effective measurement point at about $0.5 \mathrm{~mm}$ from its surface. DosiRat was calibrated in absorbed dose to water in a $4 \times 4 \mathrm{~cm}^{2}$ irradiation field at $225 \mathrm{kVp}$. It was placed at isocenter (SDD $=30.7 \mathrm{~cm}$ ), at a depth of 2 $\mathrm{cm}$ in a water tank, and irradiated at the reference dose rate (previously measured with the PTW 30013 ionization chamber, see section 2.1.1) in order to determine a calibration factor in $\mathrm{Gy} / \mathrm{pC}$.

Preclinical irradiation conditions are different from absolute dosimetry conditions, particularly in terms of beam size and scatter volume. They induce changes in the beam energy spectrum and variations of 
plastic scintillating dosimeters energy response, primarily due to the non-equivalence of the absorption coefficient of polystyrene relative to water. ${ }^{15,17}$ As detailed in Le Deroff $e t$ al work, correction factors must be calculated by Monte Carlo simulations to take into account beam energy modifications between preclinical conditions and the calibration configuration. ${ }^{17}$

The correction factors used in this work were calculated on PMMA phantom of $3 \times 9 \times 3 \mathrm{~cm}^{3}$, for dose measurements either at the beam entrance or at the beam exit with various collimator sizes (see table 1).

Table 1. DosiRat energy correction factors for dose measurement at the entrance or the exit of a $3 \times 9 \times 3 \mathrm{~cm}^{3}$ PMMA phantom, for a single anterior beam, with various collimator sizes.

\begin{tabular}{cccc}
\hline Measurement type & $25 \mathrm{~mm}$ diameter collimator & 10 mm diameter collimator & $5 \mathrm{~mm}$ diameter collimator \\
\hline Entrance & 0.984 & 0.973 & 0.969 \\
Exit & 0.957 & 0.948 & 0.945 \\
\hline
\end{tabular}

In all this study, the adequate correction factor was applied to DosiRat measurement, depending on the configuration.

\section{3. $\quad$ Treatment planning}

Phantom and rat brain irradiations were planned with a dedicated small animal TPS, SmART-Plan (Small Animal RadioTherapyPlan), developed by the MAASTRO Clinic ${ }^{12}$ and commercialized by Precision X-Ray, Inc. (North Branford, CT, USA) for the X-RAD 225Cx irradiator. The software includes the different steps of a clinical treatment planning: the upload of a CBCT acquisition, the tissues segmentation (chemical composition and density), the volumes of interest contouring and the definition of the ballistic and computation of the dose distribution. SmART-Plan is interfaced with the Monte Carlo (MC) program DOSXYZnrc ${ }^{19}$ to perform the dose computation. The dosimetric accuracy of the TPS has been assessed by the manufacturer for all the collimators through lateral and depth dose profiles and for complex irradiation plans performed in solid water. During this commissioning, computed dose distributions were compared to experimental distributions measured with radiochromic films (GafChromic 
EBT3, Ahsland Specialty Ingredients, Wayne, NJ) and showed that for the large majority of dose measurements, dosimetric agreement lied well within $5 \%$ for collimators of $5 \mathrm{~mm}$ diameter or more. This accuracy has been confirmed by radiochromic films measurements performed at the surface of our PMMA phantom and presented in the supplementary data.

The treatment planning of the phantom and rat brain irradiations performed in this work is illustrated in figure 1 and described below.
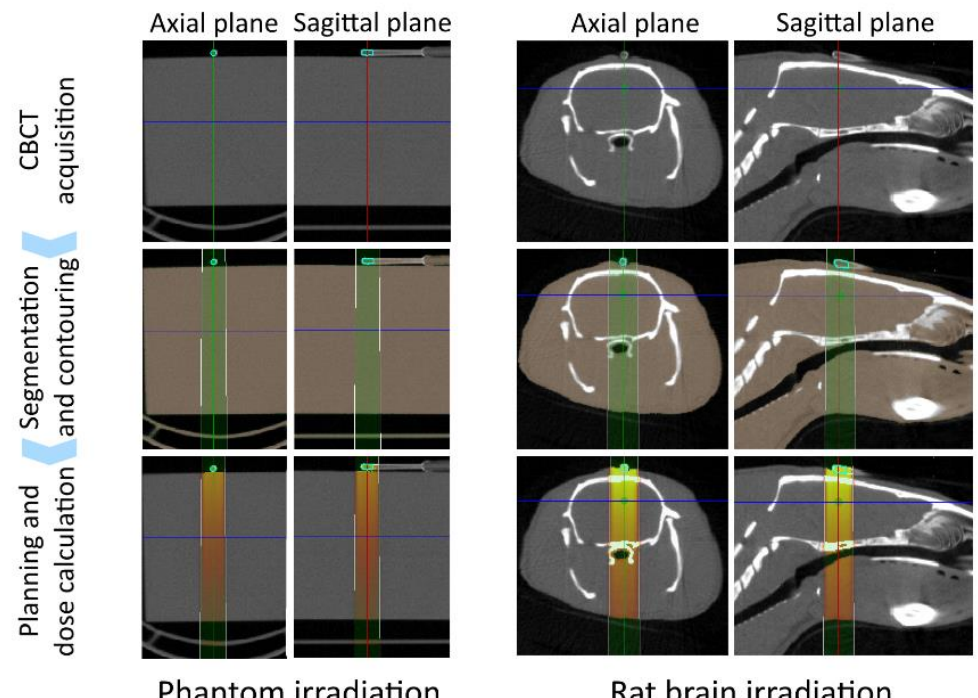

Rat brain irradiation

Figure 1. Treatment planning with DosiRat positioned at the beam entrance in a $5 \mathrm{~mm}$ diameter beam. The phantom irradiation is represented in the left side of the figure and the rat brain irradiation in the right side. In both cases, the $2 \mathrm{~mm}$ long scintillating volume is contoured in cyan and overwritten by water composition and density to correspond to the dose to water calibration. The $5 \mathrm{~mm}$ diameter irradiation beam is displayed in green in the "segmentation and contouring" step. The dose distribution produced by SmART-Plan was post-processed with ImageJ. ${ }^{21}$

A CBCT acquisition was performed at $80 \mathrm{kVp}$, with a tube current of $1 \mathrm{~mA}$, an external filtration of $2 \mathrm{~mm}$ of aluminum and a spatial resolution of $200 \mu \mathrm{m}$ in the three directions. The resulting acquisition 
was uploaded in the SmART-Plan software where the CBCT voxels were converted into the appropriate materials: PMMA and air in the case of the phantom, soft tissue, bone and air in the case of the animal irradiation. ${ }^{20}$ As DosiRat was calibrated in dose to water (see section 2.2), the scintillator volume was contoured and overwritten by water composition and density. The treatment beams were defined and aimed at the target located in the rat brain. The dose distribution was then computed with $1 \%$ statistical uncertainty, providing a dose map in DICOM format. The average dose to water in the scintillator volume was extracted from this dose map with the ImageJ analysis software. ${ }^{21}$

\subsection{Phantom irradiations}

Prior to animal experiments, the agreement between the planned and the delivered dose was evaluated with a PMMA phantom of animal size $\left(3 \times 9 \times 3 \mathrm{~cm}^{3}\right)$. CBCT acquisitions were used to accurately position DosiRat at the surface of the phantom. Two treatment configurations were planned following the steps described in section 2.3.

In the first configuration, the prescription point was defined at the phantom surface in the scintillator volume (thus positioned at a SDD of $30.7 \mathrm{~cm}$ ). An anterior or a posterior beam was planned to deliver 1 or $2 \mathrm{~Gy}$ in the scintillator, with 5 and $10 \mathrm{~mm}$ diameter collimators.

In the second configuration, the prescription point was placed at a depth of $1 \mathrm{~cm}$ below the dosimeter, to mimic the irradiation of a rat brain. An anterior or a posterior beam was planned with 5 and $10 \mathrm{~mm}$ diameter collimators to deliver $2 \mathrm{~Gy}$ at the prescription point.

\subsection{Rat brain irradiations}

Post mortem and in vivo irradiations of rat brain were planned following the procedure described in section 2.3. DosiRat was centered on each rat head prior to the treatment planning using either the positioning lasers (for the $25 \mathrm{~mm}$ diameter field irradiations as shown in figure 2) or a CBCT acquisition (for the 5 and $10 \mathrm{~mm}$ diameter irradiation fields experiments).

Post mortem irradiations were performed on 3 rats. Doses of $0.1,0.5$ and 2 Gy were prescribed in the brain with anterior beams and 5 and $10 \mathrm{~mm}$ diameter collimators. Doses of 0.1 Gy were delivered with 
a tube current of $1.3 \mathrm{~mA}$ to avoid too short irradiation times. For the doses of $0.5 \mathrm{~Gy}$, two tube currents (1.3 and $13 \mathrm{~mA}$ ) were tested. Each irradiation was reproduced three times, the animal and the dosimeter being repositioned between each measurement in order to evaluate the whole dosimetry process reproducibility.

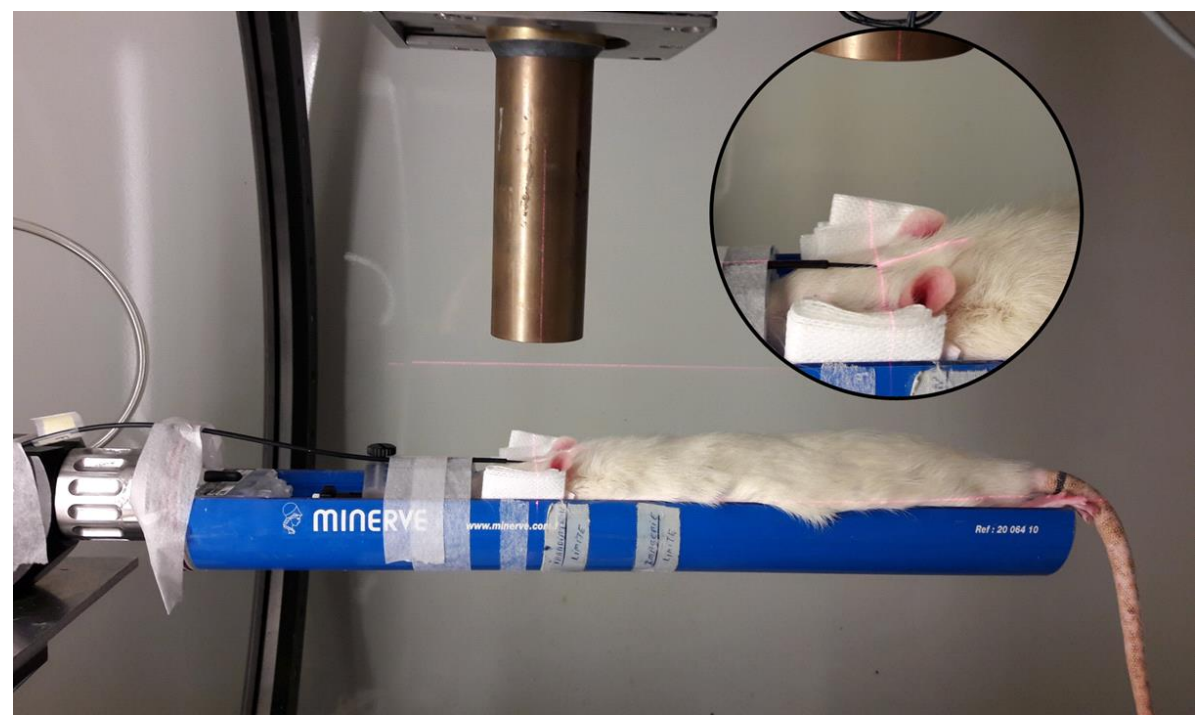

Figure 2. Irradiation of a rat brain and dose measurement by DosiRat positioned at the beam entrance, using the positioning lasers.

In vivo irradiations were performed on 6 rats. The animal investigations were performed under the current European directive (2010/63/EU) in authorized laboratories (B14118001) and with the permission of the regional and national committees on animal ethics (CENOMEXA, APAFIS\#00919). Animal care and monitoring during the experiments was insured by competent personnel for animal experimentation. Isoflurane anesthesia was used for all rats: $5 \%$ for induction and $2.5 \%$ for maintenance in a gas mixture of $70 \%$ of nitrous oxide $\left(\mathrm{N}_{2} \mathrm{O}\right)$ and $30 \%$ of oxygen $\left(\mathrm{O}_{2}\right)$. Rectal temperature was monitored and maintained around $37.0{ }^{\circ} \mathrm{C}$ throughout the experiments. A dose of $10 \mathrm{~Gy}$ was prescribed to 3 rats and a dose of $0.1 \mathrm{~Gy}$ (delivered with a tube current of $1.3 \mathrm{~mA}$ ) to the 3 other rats. The irradiations were performed with an anterior beam and a collimator of $25 \mathrm{~mm}$ diameter. 
Weight differences between post mortem and in vivo animals (about 400 and $600 \mathrm{~g}$ respectively) contributed to diversify the configurations.

\section{Results}

\subsection{Absolute dose rate and ramp-up}

A dose rate of $3.21 \mathrm{~Gy} / \mathrm{min}$ was measured in the reference irradiation conditions $(225 \mathrm{kVp}, 13 \mathrm{~mA})$, at isocenter at a depth of $2 \mathrm{~cm}$ in water, in a $4 \times 4 \mathrm{~cm}^{2}$ irradiation field. This measurement is consistent with Lindsay et al work on multi-institution commissioning of an X-RAD $225 \mathrm{Cx} .{ }^{18}$

The ramp-up effect of our irradiator was measured for tube currents of 1.3 and $13 \mathrm{~mA}$. The air kerma is presented as a function of the irradiation time in figure 3. The curves show ramp-up times of 1.9 seconds at $1.3 \mathrm{~mA}$ and 2.3 seconds at $13 \mathrm{~mA}$. These values are significant compared to typical irradiation times of approximately 40 seconds used to deliver 2 Gy, leading to an overestimation of the delivered dose by SmART-Plan. This bias was thus corrected in this entire study by subtracting the ramp-up time multiplied by the dose rate. The correction corresponds to $0.01 \mathrm{~Gy}$ at $1.3 \mathrm{~mA}$ and $0.12 \mathrm{~Gy}$ at $13 \mathrm{~mA}$.

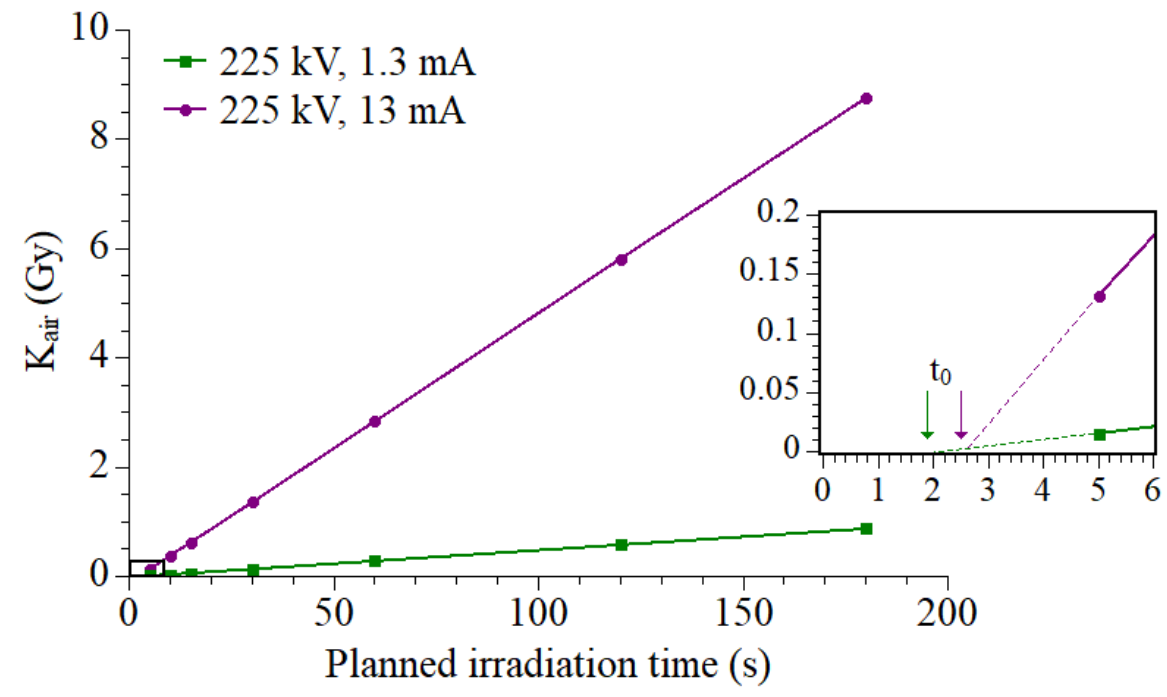

Figure 3. Ramp-up time (t0) determination with air kerma measurement expressed as a function of the irradiation time for two tube currents (1.3 and $13 \mathrm{~mA})$. The box figure is a zoomed in portion of the main figure up to $0.2 \mathrm{~Gy}$ and $6 \mathrm{~s}$. 
Additionally for the low dose-rate irradiations, the irradiator control panel indicated that the actual tube current reached by the tube was $1.335 \mathrm{~mA}$ instead of the nominal $1.3 \mathrm{~mA}$. The corresponding planned doses were thus corrected to take into account this $2.7 \%$ additional delivered dose.

\subsection{Phantom irradiations}

Table 2 presents the planned doses, the doses measured with DosiRat and the corresponding relative differences for the different irradiation configurations.

Table 2. Comparison between the planned doses and the doses measured with DosiRat at the surface of the phantom for different treatment plans. The planned dose are corrected from the ramp-up time.

\begin{tabular}{|c|c|c|c|c|c|c|}
\hline $\begin{array}{l}\text { Prescription } \\
\text { point }\end{array}$ & $\begin{array}{c}\text { Beam diam- } \\
\text { eter }(\mathrm{mm})\end{array}$ & $\begin{array}{l}\text { Prescribed dose } \\
\text { (Gy) }\end{array}$ & Ballistic & $\begin{array}{l}\text { Planned dose in } \\
\text { the dosimeter } \\
\text { (Gy) }\end{array}$ & $\begin{array}{l}\text { Measured dose } \\
\text { (Gy) }\end{array}$ & $\begin{array}{c}\text { Relative } \\
\text { difference }\end{array}$ \\
\hline \multirow{6}{*}{ DosiRat } & \multirow{3}{*}{$10 \mathrm{~mm}$} & 2 & anterior & 1.89 & 1.97 & $4.0 \%$ \\
\hline & & 1 & anterior & 0.89 & 0.91 & $2.5 \%$ \\
\hline & & 1 & posterior & 0.89 & 0.90 & $1.0 \%$ \\
\hline & \multirow{3}{*}{$5 \mathrm{~mm}$} & 2 & anterior & 1.89 & 1.96 & $3.4 \%$ \\
\hline & & 1 & anterior & 0.90 & 0.91 & $1.7 \%$ \\
\hline & & 1 & posterior & 0.89 & 0.93 & $3.4 \%$ \\
\hline & \multirow{2}{*}{$10 \mathrm{~mm}$} & 2 & anterior & 2.77 & 2.87 & $3.5 \%$ \\
\hline Phantom at & & 2 & posterior & 1.60 & 1.61 & $0.5 \%$ \\
\hline \multirow[t]{2}{*}{$1 \mathrm{~cm}$ depth } & \multirow{2}{*}{$5 \mathrm{~mm}$} & 2 & anterior & 2.87 & 2.96 & $3.2 \%$ \\
\hline & & 2 & posterior & 1.56 & 1.28 & $-17.7 \%$ \\
\hline
\end{tabular}

Discrepancies between planned and measured doses stay below 5\%, except for the last measurement which corresponds to the irradiation of a target inside the phantom with a $5 \mathrm{~mm}$ diameter posterior beam. In this configuration, even though a Winston Lutz procedure was applied prior to irradiation, ${ }^{22}$ the DosiRat sensitive volume was not entirely included in the irradiation field, leading to signal loss.

Excluding this measurement, the average difference between planned and measured doses is $2.6 \%$ with a standard deviation of $1.2 \%$. 
Table 3. Comparison between the planned doses and the doses measured with DosiRat positioned on the rat head for different treatment plans. The planned doses are corrected from the ramp-up time and the tube current actual value.

\begin{tabular}{|c|c|c|c|c|c|c|c|}
\hline $\begin{array}{l}\text { Tube } \\
\text { curent }\end{array}$ & $\begin{array}{l}\text { Beam diam- } \\
\text { eter (mm) }\end{array}$ & $\begin{array}{l}\text { Prescribed } \\
\text { dose to the } \\
\text { brain (Gy) }\end{array}$ & $\begin{array}{l}\text { Planned dose in } \\
\text { the dosimeter } \\
\text { (Gy) }\end{array}$ & $\begin{array}{l}\text { Measured dose } \\
\quad(\mathrm{Gy})\end{array}$ & $\begin{array}{l}\text { Relative } \\
\text { difference }\end{array}$ & $\begin{array}{l}\text { Average } \\
\text { difference }\end{array}$ & $\begin{array}{l}\text { Standard de- } \\
\text { viation on } \\
\text { differences }\end{array}$ \\
\hline \multirow{15}{*}{$13 \mathrm{~mA}$} & \multirow{3}{*}{25} & \multirow{3}{*}{10} & 11.16 & 11.98 & $7.4 \%$ & & \\
\hline & & & 11.06 & 11.89 & $7.6 \%$ & \multirow[t]{2}{*}{$6.5 \%$} & \multirow[t]{2}{*}{$1.7 \%$} \\
\hline & & & 11.15 & 11.66 & $4.5 \%$ & & \\
\hline & \multirow{6}{*}{10} & \multirow{3}{*}{2} & 2.31 & 2.43 & $5.3 \%$ & & \\
\hline & & & 2.24 & 2.36 & $5.5 \%$ & $5.3 \%$ & $0.2 \%$ \\
\hline & & & 2.23 & 2.35 & $5.1 \%$ & & \\
\hline & & \multirow{3}{*}{0.5} & 0.49 & 0.52 & $6.2 \%$ & & \\
\hline & & & 0.48 & 0.52 & $8.8 \%$ & \multirow[t]{2}{*}{$7.1 \%$} & \multirow[t]{2}{*}{$1.5 \%$} \\
\hline & & & 0.48 & 0.51 & $6.4 \%$ & & \\
\hline & \multirow{6}{*}{5} & \multirow{3}{*}{2} & 2.27 & 2.41 & $6.3 \%$ & & \\
\hline & & & 2.23 & 2.36 & $5.6 \%$ & \multirow[t]{2}{*}{$6.0 \%$} & \multirow[t]{2}{*}{$0.4 \%$} \\
\hline & & & 2.35 & 2.49 & $6.2 \%$ & & \\
\hline & & \multirow{3}{*}{0.5} & 0.48 & 0.49 & $1.9 \%$ & & \\
\hline & & & 0.48 & 0.51 & $6.9 \%$ & \multirow[t]{2}{*}{$5.6 \%$} & \multirow[t]{2}{*}{$3.3 \%$} \\
\hline & & & 0.50 & 0.55 & $8.1 \%$ & & \\
\hline \multirow{15}{*}{$1.3 \mathrm{~mA}$} & \multirow{3}{*}{25} & \multirow{3}{*}{0.1} & 0.10 & 0.10 & $1.8 \%$ & & \\
\hline & & & 0.11 & 0.11 & $5.0 \%$ & \multirow[t]{2}{*}{$3.3 \%$} & \multirow[t]{2}{*}{$1.6 \%$} \\
\hline & & & 0.10 & 0.11 & $3.2 \%$ & & \\
\hline & \multirow{6}{*}{10} & \multirow{3}{*}{0.5} & 0.61 & 0.61 & $-0.3 \%$ & & \\
\hline & & & 0.59 & 0.60 & $1.6 \%$ & \multirow[t]{2}{*}{$-0.7 \%$} & \multirow[t]{2}{*}{$2.5 \%$} \\
\hline & & & 0.59 & 0.57 & $-3.3 \%$ & & \\
\hline & & \multirow{3}{*}{0.1} & 0.11 & 0.12 & $3.9 \%$ & & \\
\hline & & & 0.11 & 0.12 & $2.8 \%$ & \multirow[t]{2}{*}{$3.7 \%$} & \multirow[t]{2}{*}{$0.8 \%$} \\
\hline & & & 0.11 & 0.11 & $4.4 \%$ & & \\
\hline & \multirow{6}{*}{5} & & 0.60 & 0.61 & $1.7 \%$ & & \\
\hline & & 0.5 & 0.59 & 0.64 & $8.7 \%$ & $4.2 \%$ & $3.9 \%$ \\
\hline & & & 0.62 & 0.63 & $2.3 \%$ & & \\
\hline & & & 0.11 & 0.11 & $-1.2 \%$ & & \\
\hline & & 0.1 & 0.11 & 0.11 & $-0.3 \%$ & $0.7 \%$ & $2.6 \%$ \\
\hline & & & 0.11 & 0.12 & $3.7 \%$ & & \\
\hline
\end{tabular}




\subsection{Rat brain irradiations}

Planned and measured doses obtained during post mortem and in vivo irradiations by an anterior beam are summarized in table 3. Post mortem reproducibility measurements (corresponding to the 5 and $10 \mathrm{~mm}$ diameter irradiation fields) show larger standard deviations of the relative differences when the prescribed dose is low ( 0.1 and $0.5 \mathrm{~Gy})$. On the contrary, the reproducibility does not seem to depend on the dose rate (tube current). In the case of in vivo measurements (25 $\mathrm{mm}$ diameter collimator), the standard deviations of the relative differences are comparable $(1.70$ and $1.56 \%)$ for both delivered doses. It is explained by the variability between the different animals used for each measurement and the less accurate positioning of the detector in the irradiation field with the lasers. Finally, the relative differences between planned and measured doses range from $-3.3 \%$ to $8.8 \%$ with an average value of $4.2 \%$ and a standard deviation of $3.0 \%$.

\section{Discussion}

The consistency between the planned and the measured dose obtained with the phantom (relative discrepancies ranging from 0.5 to $4.0 \%$ ) is comparable to the TPS uncertainty and other dosimetry verifications performed on phantoms with micro-irradiators. Van Hoof et $a l^{23}$ obtained a good agreement between planned doses and doses measured with EBT2 films for a plasticized heterogeneous mouse phantom (PlastiMouse - SmART Scientific solution B.V.) irradiated by a single beam with a $5 \mathrm{~mm}$ diameter collimator. Except in the penumbra area, differences were smaller than $5 \%$. Noblet et $\mathrm{al}^{24}$ compared Monte Carlo simulations performed with GATE $^{25}$ and EBT3 film measurements for a waterfilled cylinder of $2.8 \mathrm{~cm}$ diameter irradiated by a $360^{\circ}$-arc with various collimators. The doses simulated and measured on the arc rotation axis presented discrepancies of $-1.1 \%, 2.1 \%$ and $1.1 \%$ for irradiation field diameters of $20 \mathrm{~mm}, 5 \mathrm{~mm}$ and $2.5 \mathrm{~mm}$ respectively. Nevertheless, the comparison between planned and measured dose is limited by the TPS uncertainty. It doesn't allow to discriminate errors coming from the detector, the treatment planning or the delivery system. Additional investigations, such as comparison with other detectors in small fields, would thus allow to identify the different sources of error. 
Concerning in vivo dosimetry, this work presents first measurements performed with a direct-reading point detector. The comparison with planned doses shows a satisfying agreement with relative discrepancies ranging from $-3.3 \%$ to $8.8 \%$. The relative differences obtained during post mortem irradiations also seem to show larger standard deviations for the lowest doses. This effect can't be directly explained by the precision of the dosimeter as a function of the dose. Indeed Le Deroff et al ${ }^{16}$ showed an excellent repeatability of the dosimeter response at the treatment beam quality, without significant dependence with the measured dose. This observation should thus be confirmed and investigated in future experiments.

The deviations achieved in vivo are larger than the ones obtained with the phantom, which can have several explanations. Animal tissues compositions are not well known and human tissue compositions are used instead, ${ }^{9}$ which can lead to dose calculation errors. Moreover, only three different tissues and materials were used in our study: soft tissues, bone and air. Bazalova $e a^{26}$ and Noblet $e t a l^{27}$ showed that the number of tissues used to compute the dose (skin, soft tissues, brain, grey matter, white matter, fat, bones of the skull) affects the result because of the important variability of the mass absorption coefficients between the different materials in this energy range. The second possible origin of these larger differences concerns DosiRat energy response correction factors, which were computed with the PMMA phantom. The geometry of the animal head, the tissues composition and density can modify the energy spectrum and consequently induce a bias. This would be particularly true for intra-cavity or beam exit measurements. This observation raises the issue of computing individual correction factors to take into account morphological difference between animals. These factors rely on time-consuming Monte Carlo simulations (especially when they include CBCT images) which would inevitably prevent online monitoring of the delivered dose. It must be noted that this problem is relevant for any detector made from a material different from the reference materials (water or air) in kilovoltage dosimetry. ${ }^{28}$

Finally, the implementation of in vivo dosimetry at the animal scale is challenging. Besides energy variations mentioned above, the positioning of the dosimeter in millimetric beams is difficult and lasers are not accurate enough. The smallest field that could be accurately controlled in this work was $5 \mathrm{~mm}$ di- 
ameter. This could be improved with a smaller scintillating probe. The dosimeter positioning reproducibility and the impact of positioning errors on dose reading should also be investigated to determine the reliability of the lasers depending on the beam size. When an accurate positioning can't be achieved with the lasers, CBCT imaging allows sub-millimeter accuracy to align the dosimeter with the beam axis but attention must be given to the additional dose delivered during positioning imaging. Indeed, air kerma measurements and MC simulations showed that the CBCT acquisitions performed in this study deliver about $0.06 \mathrm{~Gy}$ to the phantom. This value is significant relative to planned doses of $0.1 \mathrm{~Gy}$ and should be considered during the interpretation of biological results. The imaging panel could also be used in conjunction with the irradiation collimator to quickly determine if the active volume of the scintillator is in the irradiation field, which should limit the imaging dose delivered to the animal. Twodimension detectors such as dosimetry films avoid this difficulty. Nevertheless, they are not adapted to control the beam position relative to the irradiation target, preventing the detection of positioning errors or motions during irradiation.

\section{Conclusion}

This work highlighted the complexity to implement in vivo dosimetry in orthovoltage millimetric beams. The plastic scintillating detector DosiRat is a suitable dosimeter that showed good results to answer this issue. In vivo measured dose showed discrepancies with planned doses smaller than $8.8 \%$ for anterior beams down to $5 \mathrm{~mm}$ diameters. Despite the small size of DosiRat sensitive volume, the dosimetry of smaller beams is limited by the positioning of the detector on the beam axis. Thanks to its size and its direct reading, DosiRat has the potential to perform online dose monitoring.

\section{References}

1. F. Verhaegen, P. Granton and E. Tryggestad, Small animal radiotherapy research platforms. Phys Med Biol. 2011;56:R55. doi: 10.1088/0031-9155/56/12/R01

2. K. T. Butterworth, K. M. Prise and F. Verhaegen, Small animal image-guided radiotherapy : status, considerations and potential for translational impact. The British Journal of Radiology. 2015; 88(1045):20140634. doi: 10.1259/bjr.20140634

3. M. Desrosiers, L. DeWerd, J Deye et al., The Importance of Dosimetry Standardization in Radiobiology. J Res Nat Inst Stand Technol. 2013;118:403-418. doi: 10.6028/jres.118.021 
4. Pedersen KH, Kunugi KA, Hammer CG, Culberson WS, DeWerd LA. Radiation biology irradiator dose verification survey. Radiat Res.2016;185:163-168.

5. Ngwa W, Korideck H, Chin LM, Makrigiorgos GM, Berbeco RI. MOSFET assessment of radiation dose delivered to mice using the small animal radiation research platform (SARRP). Radiat Res. 2011;176:816-820. PMID: 21962005

6. A. Anvari, Y. Poirier and A. Sawant, Development and implementation of EPID-based quality assurance tests for the small animal radiation research platform (SARRP). Med. Phys. 2018;45( 7):3246-3257. doi: 10.1002/mp.12939

7. P. Kuess, E. Bozsaky, J. Hopfgartner, G. Seifritz, W. Dorr and D. Georg, Dosimetric challences of small animal irradiation with a commercial X-ray unit. Z Med. Phys. 2018; 24(4):363372. doi: 10.1016/j.zemedi.2014.08.005

8. INTERNATIONAL ATOMIC ENERGY AGENCY. Development of Procedures for In Vivo Dosimetry in Radiotherapy. No. 8 in IAEA Human Health Reports. Vienna: INTERNATIONAL ATOMIC ENERGY AGENCY; 2013. http://www-pub.iaea.org/books/IAEABooks/8962/ Development-of-Procedures-for-In-Vivo-Dosimetry-in-Radiotherapy.

9. P. V. Granton, M. Podesta, G. Landry, S. Nijsten, G. Bootsma and F. Verhaegen, A combined dose calculation and verification method for a small animal precision irradiator based on onboard imaging. Med. Phys. 2012;39(7):4155-4166. doi: 10.1118/1.4725710

10. L Beaulieu, M Goulet, L Archambault and S Beddar. Current status of scintillation dosimetry for megavoltage beams. J Phys Conf Ser. 2013;444(1):012013.

11. Beddar AS. Plastic scintillation dosimetry and its application to radiotherapy. Radiat Meas. 2006;41, Supplement 1(0):S124 \{ S133. The 2nd Summer School on Solid State Dosimetry:

Concepts and Trends in Medical Dosimetry. http://www.sciencedirect.com/science/article/pii/S1350448707000108.

12. P. Carrasco, N. Jornet, O. Jordi et al, Characterization of the Exradin W1 scintillator for use in radiotherapy. Med. Phys. 2015;42(1):297-304. doi: 10.1118/1.4903757

13. D. M. Klein, R. C. Tailor, L. Archambault, F. Therriault-Proulx and A. S. Beddar, Measuring output factors of small fields formed by collimator jaws and multileaf collimator using plastic scintillation detectors. Med. Phys. 2010;37(10):5541-5549. doi: 10.1118/1.3488981

14. J. Morin, D. Béliveau-Nadeau, E. Chung et al., A comparative study of small field total scatter factors and dose profiles using plastic scintillation detectors and other stereotactic dosimeters : The case of the CyberKnife. Med. Phys. 2013;40(1):011719-11. doi: $10.1118 / 1.4772190$

15. C. Johnstone, F. Therriault-Proulx, L. Beaulieu and M. Bazalova-Carter, Characterization of a Plastic Scintillating Detector for the Small Animal Radiation Research Platform (SARRP). Med Phys. 2018;46(1):394-404. doi: 10.1002/mp.13283

16. C. Le Deroff, A.-M. Frelin-Labalme and X. Ledoux, Characterization of a scintillating fibre detector for small animal imaging and irradiation dosimetry. Br J Radiol. 2017;90(1069):20160454. doi: 10.1259/bjr.20160454 
17. C. Le Deroff, A. Frelin and X. Ledoux, Energy dependence of a scintillating fiber detector for preclinical dosimetry with an image guided micro-irradiator. Phys Med Biol. 2019;[Published online on April the 11, 2019]. doi: 10.1088/1361-6560/ab1854

18. P. E. Lindsay, P. V. Granton, A. Gasparini et al, Multi-institutional dosimetric and geometric commissioning of image-guided small animal irradiators. Med. Phys. 2014;41(3):03171411. doi: 10.1118/1.4866215.

19. B. Walters, I. Kawrakow and D.W.O. Rogers, DOSXYZnrc Users Manual. NRCC Report PIRS794revB. 2010.

20. D. R. White, I. J. Wilson, J. Booz, J. J. Spokas and R. V. Griffith, Report 44, Journal of the International Commission on Radiation Units and Measurements. 1989;1:.

21. C. A. Schneider, W. S. Rasband and K. W. Eliceiri, NIH Image to ImageJ: 25 years of image analysis. Nat Methods. 2012;9(7):671-5. PMCID: PMC5554542

22. W. Lutz, K. R. Winston and N. Maleki, A system for stereotactic radiosurgery with a linear accelerator. Int J Radiat Oncol Biol Phys. 1988;14(2):373-81. doi: 10.1088/00319155/61/9/3521

23. S. J. van Hoof, P. V. Granton and F. Verhaegen, Development and validation of a treatment planning system for small animal radiotherapy : SmART-Plan. Radiother Oncol. 2013;109(3): 361-366. doi: 10.1016/j.radonc.2013.10.003

24. D. Sarrut, M. Bardiès, N. Boussion et al, A review of the use and potential of the GATE Monte Carlo code for radiation therapy and dosimetry applications. Med. Phys. 2014;41(6):064301. doi: 10.1118/1.4871617

25. C. Noblet, S. Chiavassa, F. Smekens et al, Validation of fast Monte Carlo dose calculation in small animal radiotherapy with EBT3 radiochromic films. Phys Med Biol. 2016;61(9):3521-35. doi:

26. M. Bazalova and E. E. Graves, The importance of tissue segmentation for dose calculations for kilovoltage radiation therapy. Med. Phys. 2011;38(6):3039-49. doi: 10.1118/1.3589138

27. C. Noblet, S. Chiavassa, F. Paris, S. Supiot, A. Lisbona and G. Delpon, Underestimation of dose delivery in preclinical irradiation due to scattering conditions. Phys. Med. 2014;30(1):638. doi: 10.1016/j.ejmp.2013.03.001

28. R. Hill, B. Healy, L. Holloway, Z. Kuncic, D. Thwaites and C. Baldock. Advances in kilovoltage x-ray beam dosimetry. Phys Med Biol. 2014;59(6):R183-231. doi: 10.1088/00319155/59/6/R183

29. Hubbell J and Seltzer S. Tables of X-Ray Mass Attenuation Coefficients and Mass EnergyAbsorption. 19 Juillet 2019. [On line]. Available: https://www.nist.gov/pml/x-ray-massattenuation-coefficients.

30. Attix FH.Introduction to radiological physics and radiation dosimetry. Wiley;1986.

31. Micke A, Lewis DF and Yu X. Multichannel film dosimetry with non uniformity correction. Med Phys. 2011;38(5):2523-2534. doi:10.1118/1.3576105.

\section{Acknowledgements}


This work was supported by the French National Agency for Research $n^{\circ}$ ANR 10-EQPX-0014 and Cancéropôle Nord Ouest (Emergence grant).

\section{Conflict of interest:}

The authors declare no conflict of interest. 